\title{
Arm Cortex S3C2440 Microcontroller Application for Transcranial Magnetic Stimulation's Pulse Forming on Bax Reactive Cells and Cell Death in Ischemia Induced Rats
}

\author{
Han-Ho Tac ${ }^{1}$ and Whi-Young Kim ${ }^{2 *}$ \\ ${ }^{1}$ Department of Electrical Engineering, Gyeongnam National University of Science and Technology \\ ${ }^{2}$ Department of Biomedical Engineering, Dongju College, 15-1 San, Koijeng-dong, Saha-gu, Busan 49318, Korea
}

(Received 21 January 2016, Received in final form 24 March 2016, Accepted 24 March 2016)

\begin{abstract}
Transcranial magnetic stimulation devices has been used mainly for diagnostic purposes by measuring the functions of the nervous system rather than for treatment purposes, and has a problem of considerable energy fluctuations per repeated pulse. The majority of strokes are caused by ischemia and result in brain tissue damage, leading to problems of the central nervous system including hemiparesis, dysfunction of language and consciousness, and dysfunction of perception. Control is difficult and the size is large due to the difficulty of digitalizing the energy stored in a capacitor, and there are many heavy devices. In addition, there are many constraints when it is used for a range of purposes such as head and neck diagnosis, treatment and rehabilitation of nerve palsy, muscle strengthening, treatment of urinary incontinence etc. Output stabilization and minimization of the energy variation rate are required as the level of the transcranial magnetic stimulation device is dramatically improved and the demand for therapeutic purposes increases. This study developed a compact, low cost transcranial magnetic stimulation device with minimal energy variation of a high repeated pulse and output stabilization using a real time capacitor charge discharge voltage. Ischemia was induced in male SD rats by closing off the common carotid artery for 5 minutes, after which the blood was re-perfused. In the cerebrum, the number of PARP reactive cells after 24 hours significantly decreased $(p<0.05)$ in the TMS group compared to the GI group. As a result, TMS showed the greatest effect on necrosis-related PARP immuno-reactive cells 24 hours after ischemia, indicating necrosis inhibition, blocking of neural cell death, and protection of neural cells.
\end{abstract}

Keywords : transcranial, magnetic, pulse, BAX, stimulation, treatment, cell

\section{Introduction}

Neurons are stimulated when a voltage pulse or current pulse is flowed to neurons [2]. Electric nerve stimulation system using this phenomenon is used widely for diagnostic and therapeutic purposes [3,5]. When neurons are exposed to an electric pulse using an Electrical Nerve Stimulation System, the surface electrodes should be attached to the skin of living bodies or needle electrodes should be inserted inside the living bodies [2]. There is a problem that the skin is damaged due to the current clustered around the electrodes [3-5]. To solve these problems, a magnetic nerve stimulation system was developed to replace a considerable part of the Electrical Nerve

CThe Korean Magnetics Society. All rights reserved.

*Corresponding author: Tel: +82-51-200-3235

Fax: +82-51-200-3449, e-mail: ndyag@hanmail.net
Stimulation System [1, 6, 7]. Magnetic nerve stimulation systems have evolved considerably since it was developed for the first time in the early 1980s. Magnetic nerve stimulation system employs the phenomenon that a magnetic field varying with time is flowed to living bodies and an electric field is induced inside living bodies $[8,9]$. Since the size of electric field induced should be the size of the electric field used in electric stimulation, i.e. approximately tens $\sim$ hundreds of $\mathrm{V} / \mathrm{m}$, the size pulse type time varying magnetic field (time varying magnetic field) to be flowed to living bodies should be approximately tens of Tesla and the switching time of the time varying magnetic field should be less than hundreds of $\mu \mathrm{Sec}$. Because of these technical constraints to switch a strong magnetic field within a short time, magnetic field pulse occurrence frequency for magnetic nerve stimulation system in the early stage was less than several $\mathrm{Hz}[10$, 11]. Therefore, the Magnetic Nerve Stimulation System 
has been used primarily for diagnostic purposes to measure the functions of the nerve system rather than for therapeutic purposes. Owing to recent advances in power electronics technology, the magnetic field pulse occurrence frequency of magnetic nerve stimulation system was improved to the level of $20-50 \mathrm{~Hz}$ [9]. When a Stimulation System is used for therapeutic purpose, such as rehabilitation of nerve palsy, muscle strengthening, treatment of urinary incontinence etc., $5-50 \mathrm{~Hz}$ of lowfrequency band is used frequently for the frequency of electric pulse. As the magnetic field pulse frequency of the magnetic nerve stimulation system improves to a level comparable to the electrical pulse frequency of the Electrical Stimulation System, the possibility of employing a magnetic nerve stimulation system for therapeutic purposes has attracted considerable attention. Minimization of the energy fluctuations and output stabilization are required for therapeutic purposes due to the dramatic improvement in transcranial magnetic stimulation $[1,11]$. This study developed a compact, low cost transcranial magnetic stimulation device with a minimal energy variation rate of the high repeated pulse and output stabilization by monitoring the capacitor charge voltage.

\section{Methods}

\subsection{Research model}

To stimulate the nerves magnetically, the size of the electric field induced by the time varying magnetic field should be at the level to stimulate the nerves. For this, an electric field tens of $\mathrm{V} / \mathrm{m}$ or more can be induced at the nerve part. To induce an electric field with the size of this

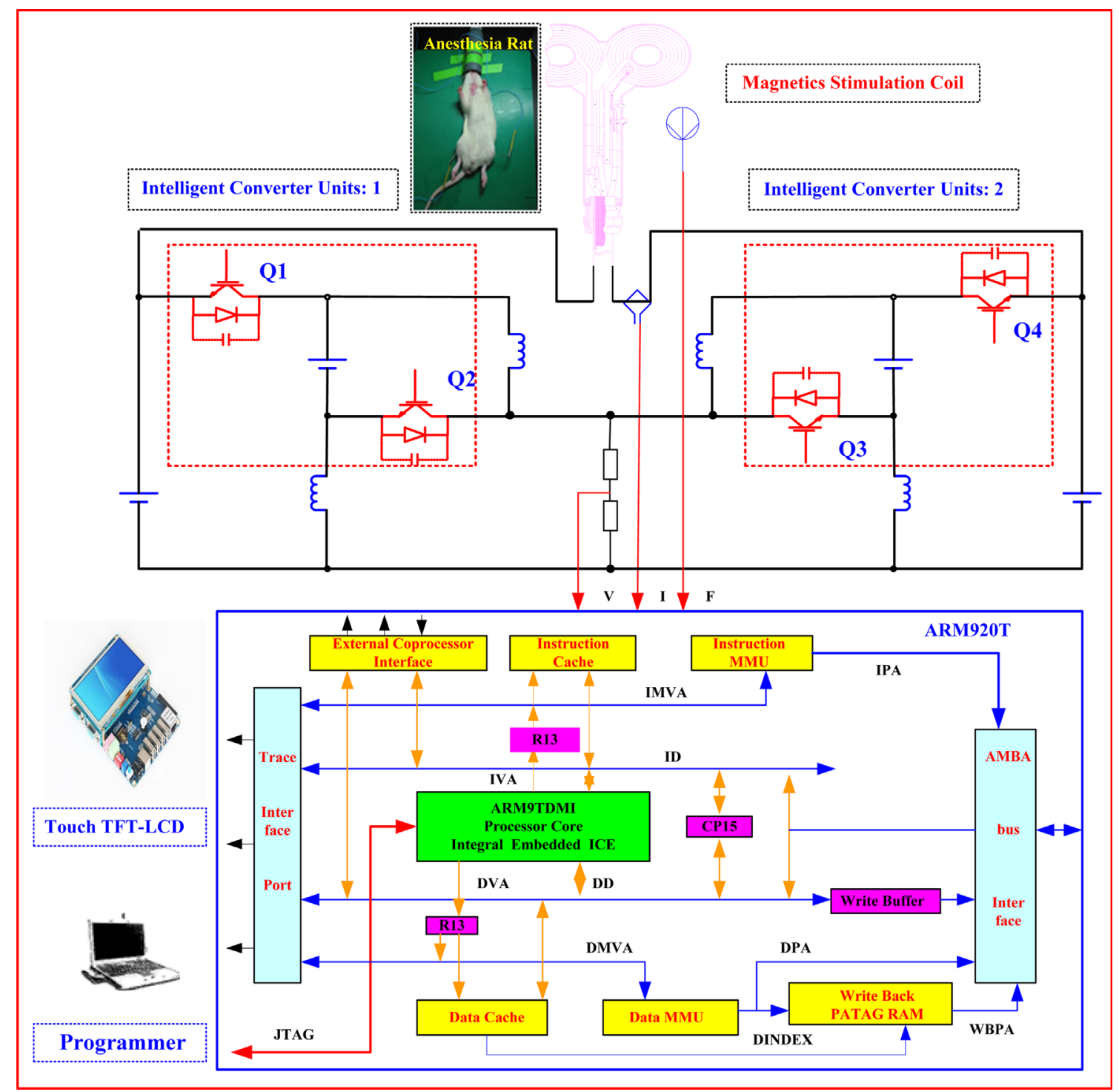

Fig. 1. (Color online) Diagram of the proposed transcranial magnetic-stimulation device. 
level, although it differs according to the distance to the place where there are nerves from the epidermis and shape of the stimulating coil, a magnetic field of 1-2Tesla should be switched within a short time of approximately $100 \mu \mathrm{sec}$. To create a magnetic field, a current of thousands of A should be flowed instantaneously to the stimulating coil, whose diameter is approximately $10 \mathrm{~cm}$ and there are dozens of coils. As a way of flowing large currents to the stimulating coil instantaneously, the capacitor is generally charged with an electric charge and discharge to stimulate the coil temporarily. Since the magnetic stimulating coil is shown as the sum of the inductance and resistance in the circuit, a capacitor and stimulating coil form a RLC resonant circuit, as shown in Fig. 1. In Fig. 1, a switch between the capacitor and stimulating coil normally consists of an IGBT (Insulate Gate Bipolar Transistor) with high internal pressure and high current capacity. Figure 1 diagram of the proposed transcranial magnetic-stimulation device. Therefore, if an electric charge begins to charge to the stimulating coil from a capacitor by opening the IGBT after charging an electric charge to a capacitor, the current waveform flowing through the stimulating coil will be determined by the RLC resonance. The vector field quantity that is related to the electric current is the time-varying current density, which is measured in amperes per square meter.

$$
\begin{aligned}
& i=\iint_{s} \vartheta \cdot d s(A) \\
& i=\iint_{s} \vartheta \cdot d s=\frac{-d q}{d t}
\end{aligned}
$$

The power consumption of a Magnetic Stimulation device can be expressed as follows:

$$
P=\frac{f_{\text {train }}(\Delta W c)}{\eta}
$$

where $f_{\text {train }}$ is the pulse train frequency, $\Delta W c$ is the energy dissipated per pulse and $\eta$ is the capacitor charger efficiency. As shown in Figs. 1, 2 back pressure power circuits were made using a capacitor for a power split and was operated as a half-bridge type by adding a resonant circuit. At this time, the on/off operation of a switch was set to occur when the current flowing through the switch was 0 . Therefore, there was little loss due to switching. Switches I-1 and I-2 are turned on and off sequentially according to resonant frequency of the current.

\subsection{Magnetic stimulation device of pulse forming ap- proach}

As a switching element, an IGBT (Insulated Gate Bipolar Transistor) suitable for some $\sim$ tens of $\mathrm{kHz}$ was used. The IGBT a hybrid semiconductor element combines the high power characteristics of the BJT with the high speed switching characteristics of the power MOSFET. Moreover, it is easier to operate than BJT since input impedance between the ammeters and gate as a voltage control element are quite high, like a MOSFET. Like the BJT, because the on-drop is low regardless of the current and is almost constant, it can flow a larger current than a MOSFET and has the characteristics of reverse voltage blocking. $C_{R}$ was used as a blocking capacitor. In an operating Half-bridge converter, in the case that input capacitors $\mathrm{C}_{1}, \mathrm{C}_{2}$ do not halve the input voltage Vi precisely or the On time of switches I-1, I-2 do not match exactly, the transformer may be saturated by causing an imbalance in the changes of in the magnetic flux of the transformer. By adding a blocking capacitor between the input voltage and transformer, the DC component of the transformer voltage causing an imbalance in the magnetic flux change can be removed. The desired input energy is passed through the bridge diode via the transformer. Therefore, it can be used in the form of a pulse by charging the desired energy to the loaded capacitor. Basically, the A/D converter is built in an Samsung 32bits S3C2440 microprocessor and has an advantage of a large current operating capability of input/output port. Therefore, the circuit configuration can be simplified. The A/D converter is the control circuit using an Samsung 32bits S3C2440 microprocessor and the value charged in a capacitor should be kept constant to maintain a constant magnetic stimulation device output. In addition, it recognizes the signal in the I-1 and I-2 in the beginning and reads the voltage charged to the capacitor and inputs this value to 32bits Arm cortex S3C2440 Microcontroller through low pass filter (LPF).

This value is then displayed in real time on a character LCD, and is treated through the AVR and the SCR signal is recognized. Figure 2, Induction of anesthesia and fixation of experiment animal using $2 \%$ isoflurane. Figure

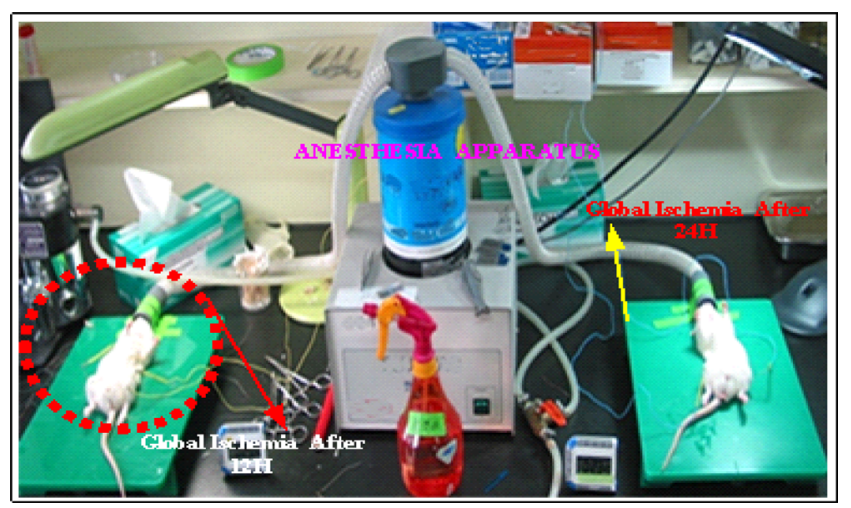

Fig. 2. (Color online) Induction of anesthesia and fixation of experiment animal using $2 \%$ isoflurane. 


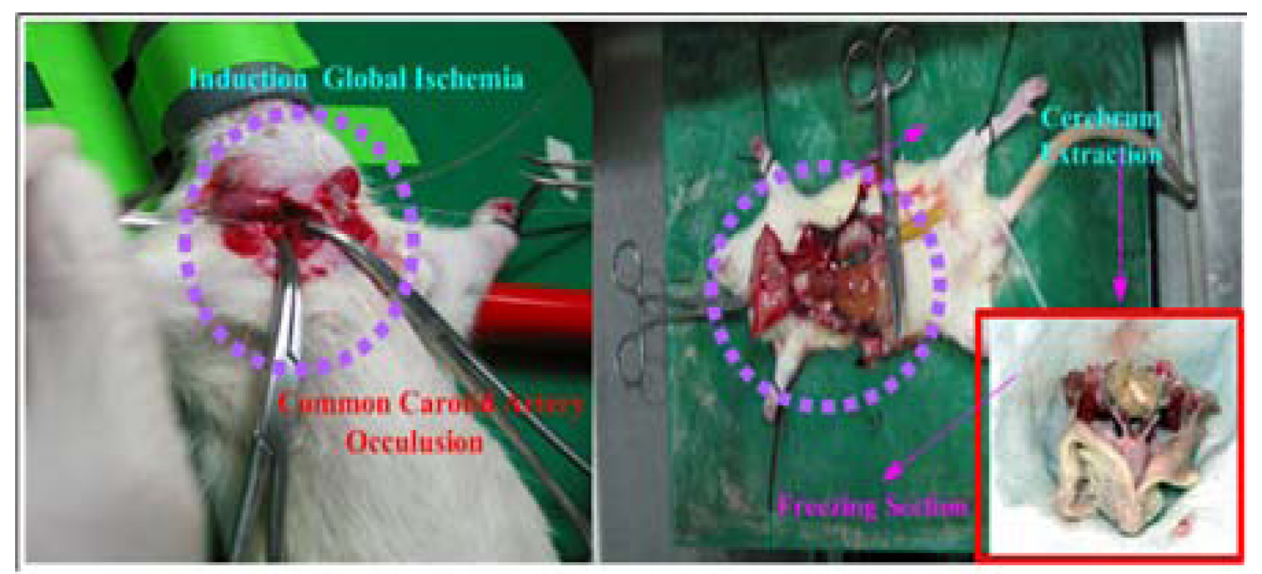

Fig. 3. (Color online) Induction of global ischemia through common carotid artery occlusion after induction of anesthesia.

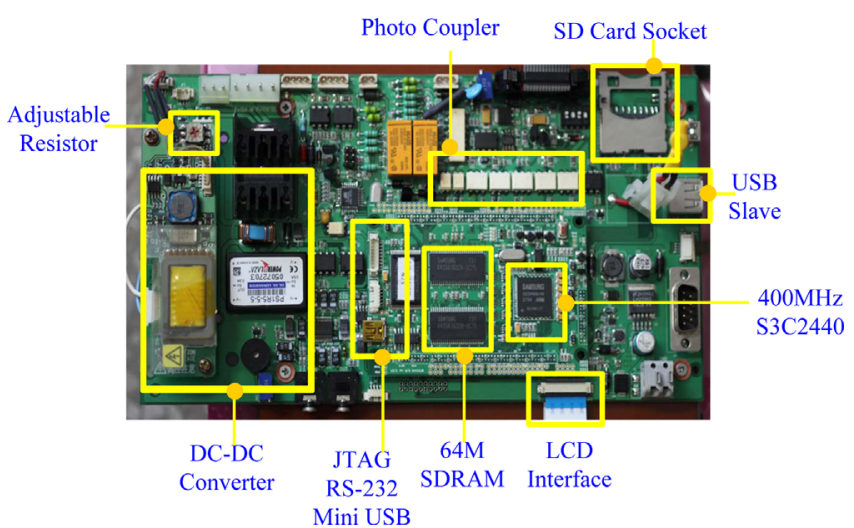

Fig. 4. (Color online) S3C 2440 chip control and drive circuit.

3 Induction of global ischemia through common carotid artery occlusion after induction of anesthesia. Figure 4
Shows the circuit to configure the 32bits Arm cortex S3C2440 Microcontroller and perform the IGBT operation and various controls. Each figure shows that when the signal by the ADC has a value of 10[V], the IGBT operating signal is stopped and the trigger signal of IGBT is recognized with a $1 \mathrm{mS}$ delay time. Figure 4 Induction of global ischemia through common carotid artery occlusion after induction of anesthesia. Figure 5 Waveforms of Primary Transcranial magnetic stimulation.

\subsection{Data Analysis}

All the collected data was analyzed by a statistical treatment Program (SPSS 12.0K/PC) after coding. After inducing apoplexy, the analysis between the treatment groups was carried out. ANOVA analysis was conducted to examine the treatment effects. The significance level was set to $\alpha=0.05$.

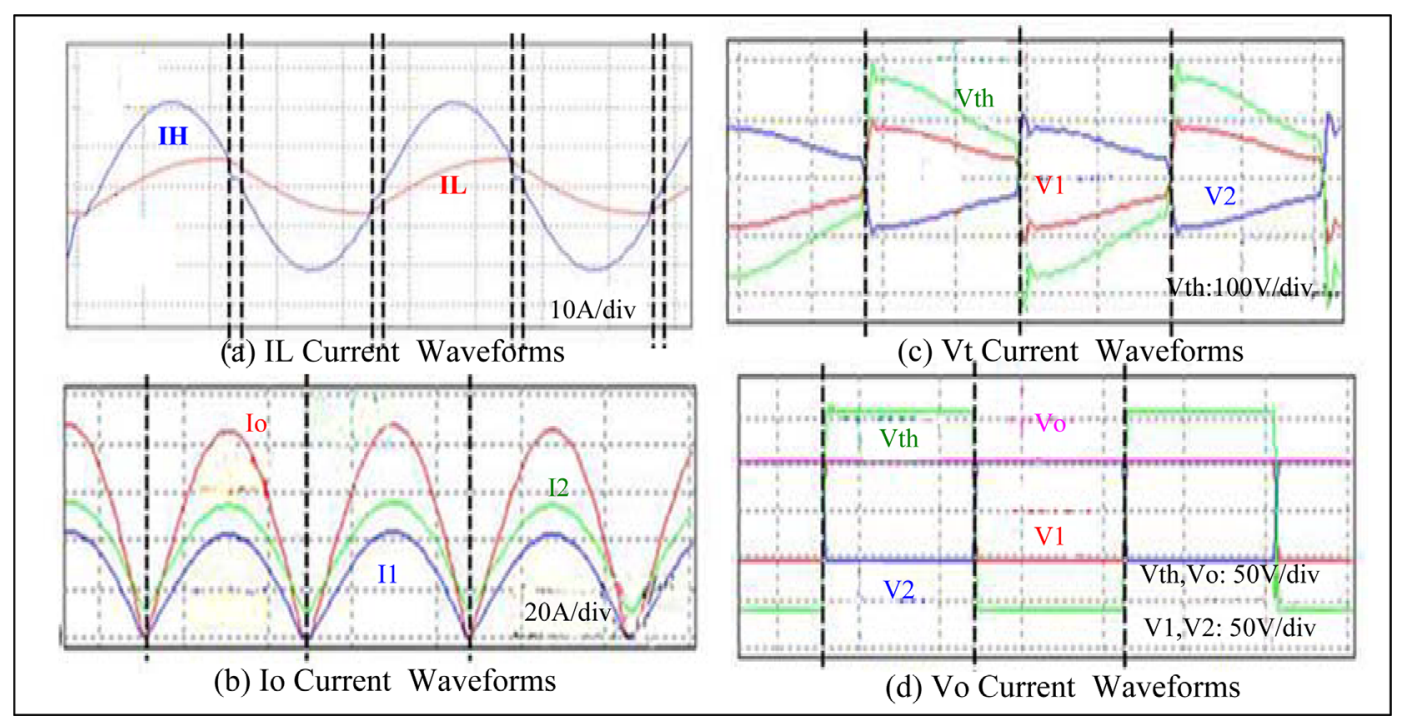

Fig. 5. (Color online) Waveforms of Primary Transcranial magnetic stimulation. 


\section{Results}

In this experiment, the load capacitor and control device as a photocoupler was insulated and used as the input value. Operation of the system was determined by reading the input value using a character LCD and treating it in the AVR in real time. The current and voltage were measured using a high-voltage probe (Probe X 6,000 V, Lecroy) and a Rogoski current waveform transducer (CWT, Penmuk). Figure 2, It is listed by site stimulation coil with the actual design. Figure 4 We show how to implement the various experiments. 32bits Arm cortex S3C2440 Microcontroller IGBT (I-1, I-2, I-3) operating signal. Initially, since the voltage of the capacitor of the sub-bottom is zero, considerable resonance current flows but resonant current tends to decrease as a capacitor is charged gradually. If it reaches the setting value, the current is reduced to zero. Operation of IGBT is ended and the trigger signal of the IGBT of the $2^{\text {nd }}$ side is approved, and the operation is switched from the capacitor charge mode to the capacitor discharge mode. By giving a $1 \mathrm{mS}$ delay time to the complete operation, operation of the half bridge converter is finished and goes into discharge mode of the $2^{\text {nd }}$ side. Figure 6 Experimental Converter Waveforms for TMS. Inverter output current, charging current and IGBT drive signals. Figure experimental waveforms for Inverter output current, IGBT and SCR drive signals. The current waveform of the $2^{\text {nd }}$ side transformer caused by interlinking with the magnetic flux by the current of the $1^{\text {st }}$ side. This waveform appears similar to the resonant current waveform of the $1^{\text {st }}$ side. Initially, a certain size of the current waveform is shown for the energy charge but as energy charge is completed, the peak of the current waveform becomes smaller. Since the IGBT is operated in the on-off cycle 50[ $\mu \mathrm{s}]$, the frequency when charging a capacitor is operated in maximum $5[\mathrm{kHz}]$.

\section{Discussions}

Figure 6, shows the discharge current waveform of the stimulating coil using the current C.T. when the magnetic stimulation device constituted a continuous blast and the waveform of the stimulating coil is constant. When the waveform of the stimulating coil was expanded to $50[\mu \mathrm{s}]$, the half amplitude of this waveform was approximately $150[\mu \mathrm{s}]$. It shows the results of simulation (Maxwell 3D: Coil whole B vector, B magnitude) Helix Type Coil analysis of the stimulation coil with the ANSOFT Tool. It shows the results of simulation (Maxwell 3D: Coil whole B vector, B magnitude) Helix Type Coil analysis of the stimulation coil with the ANSOFT Tool. Figure presents the measured proposed circuit and transcranial magnetic stimulation device output value of the general control method. Figure 7 After Stimulating pulse from fMRIFDG PET-RAT, Stimulating coil voltage waveform in a repetition pulse. When comparing the progress of stimulating coil output, this shows an approximately $15 \%$ change rate.

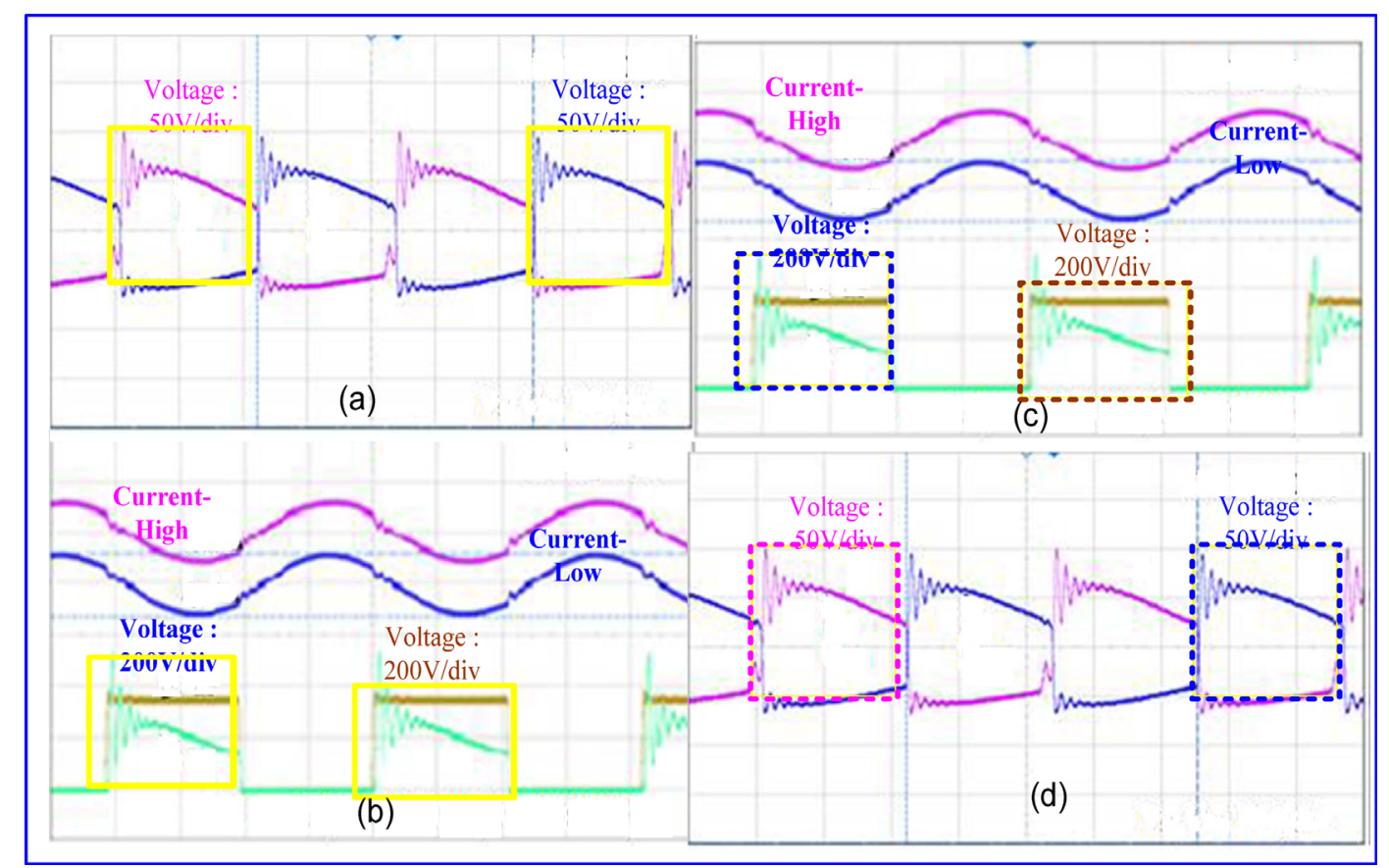

Fig. 6. (Color online) Experimental Converter Waveforms for TMS. 


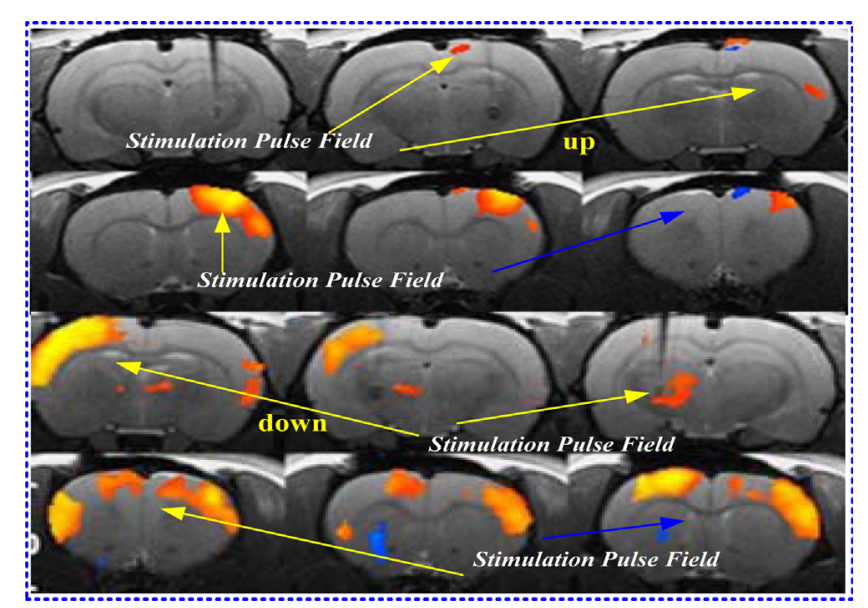

Fig. 7. (Color online) After Stimulating pulse from fMRIFDG PET-RAT.

A resulting difference in output of energy will occur when rectifying at the high pressure side first, and the load capacitor is charged through the IGBT switch, inductor, current stable resistance at the power of the smoothing capacitor. Since there is no accurate measurement of the input energy of the load capacitor, it is impossible to store the precise amount of energy. In the case of the proposed circuit, storage of the energy digitized due to the introduction of a Feedback system, which can accurately measure the energy of the load capacitor, may be possible and in this way, stabilization of the output can be achieved. The stability of the output on each energy was quite stable and efficient. In the actual graph, if the output is low, there appears to be no energy fluctuations but a larger error can be observed with small fluctuations because the total average output is low. When the load capacitor input voltage was $1,700[\mathrm{~V}]$, a larger output fluctuation was observed outwardly but showed smaller fluctuations than when the input voltage was 1,400 and 1,450[V]. Figure 8, After Stimulating pulse from fMRIFDG PET-RAT. Effect of transcranial magnetic stimulation on PARP immunore active cells in rat cerebrum after transient global ischemia. (a) Control = no surgical procedure (X 50), (b) Control = no surgical procedure (X 250), (c) $\mathrm{GI}=12 \mathrm{~h}$ after transient global ischemia (X 50), (d) $\mathrm{GI}=12 \mathrm{~h}$ after transient global ischemia (X 250), (e) $\mathrm{GI}=24 \mathrm{~h}$ after transient global ischemia (X 50), (f) $\mathrm{GI}=24 \mathrm{~h}$ after transient global ischemia (X 250), (g) $\mathrm{GI}=48 \mathrm{~h}$ after transient global ischemia (X 50), (h) $\mathrm{GI}=48 \mathrm{~h}$ after transient global ischemia (X 250), (i) TMS $=$ transcranial magnetic stimulation at $12 \mathrm{~h}$ after transient global ischemia (X 50), (j) TMS = transcranial magnetic stimulation at $12 \mathrm{~h}$ after transient global ischemia (X 250), (k) TMSS = transcranial magnetic stimulation at $24 \mathrm{~h}$ after transient global ischemia (X 50), (1) TMS = transcranial magnetic stimulation at $24 \mathrm{~h}$ after transient global ischemia (X 250), (m) TMS = transcranial magnetic stimulation at $48 \mathrm{~h}$ after transient global ischemia (X 50), (n) $\mathrm{TMS}=$ transcranial magnetic stimulation at $48 \mathrm{~h}$ after transient global ischemia (X 250). Figure 9, Bax reaction in the forebrain.
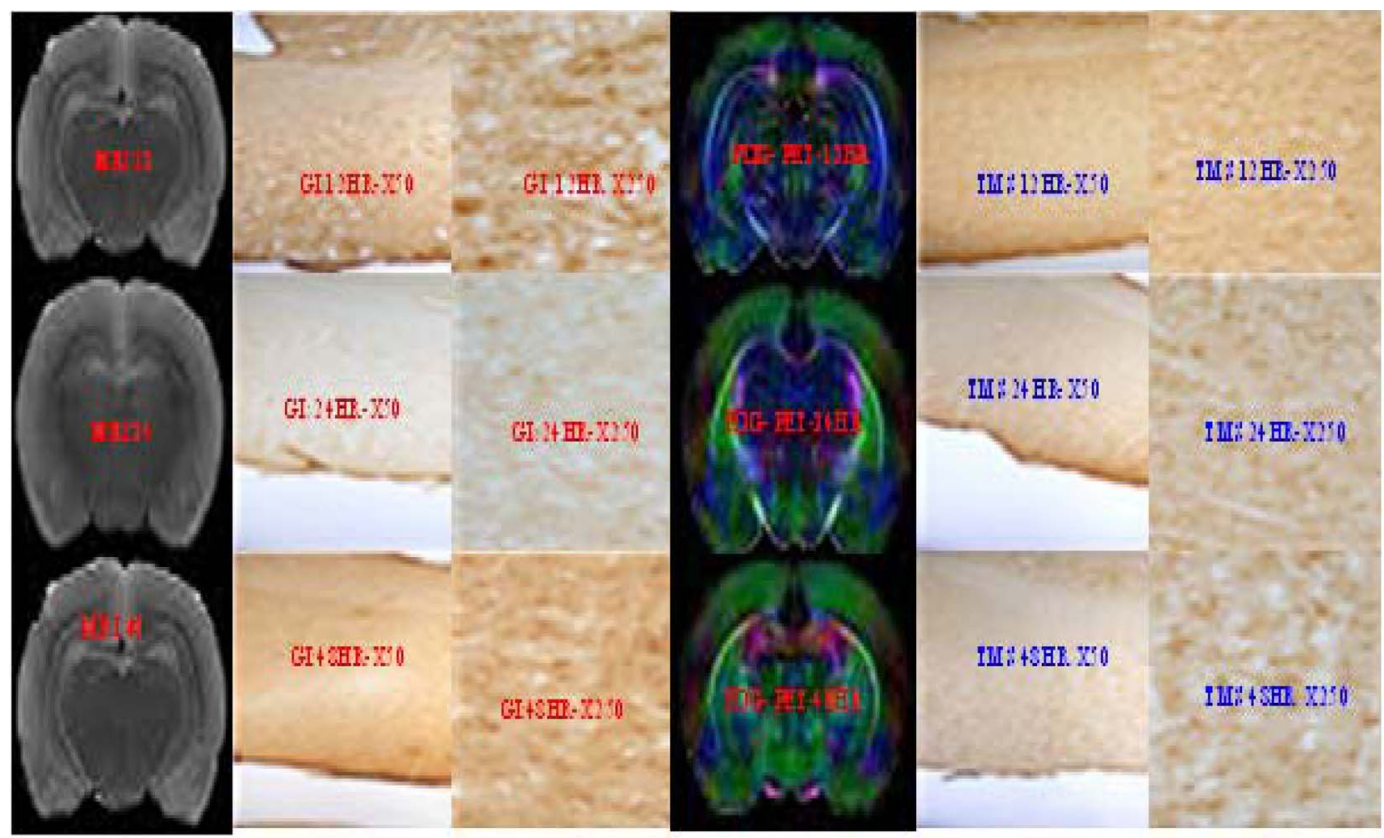

Fig. 8. (Color online) Bax reaction in the forebrain. 


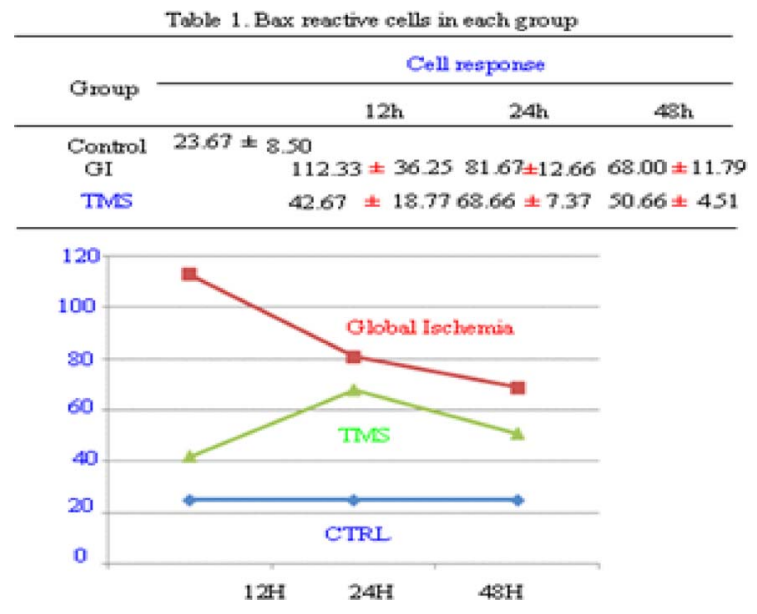

Fig. 9. (Color online) Bax reaction in the forebrain.

\section{Conclusion}

A half-bridge resonant converter and 32bits Arm cortex S3C2440 Microcontroller were used to ensure the stability of output by controlling the charge voltage of a capacitor storing input energy. The output stabilization of magnetic stimulation device, monitoring charge voltage in a capacitor was investigated. A precise value was charged through the Feedback, the charging capacitor was charged to $1,400-1,700[\mathrm{~V}]$ using a real time monitoring format with a one chip microprocessor. The Magnetic Stimulation device output per pulse according to charge voltage was within $4 \%$ tolerance, which is a greatly improved value compared to the approximately $15 \%$ output tolerance of the existing device, particularly in case of $1,600[\mathrm{~V}]$, where the output by a single pulse had a $20 \%$, minimum value. By introducing the Feedback system by monitoring the capacitor charge voltage with the existing system, the current waveform was found to be reliable in a single and continuous pulse. Ischemia was induced in male SD rats by closing off the common carotid artery for 5 minutes, after which the blood was re-perfused. In the cerebrum, the number of PARP reactive cells after 24 hours significantly decreased $(p<0.05)$ in the TMS group compared to the GI group. As a result, TMS showed the greatest effect on necrosis-related PARP immuno-reactive cells 24 hours after ischemia, indicating necrosis inhibition, blocking of neural cell death, and protection of neural cells.

\section{Acknowledgements}

"This work was supported by Gyeongnam National University of Science and Technology Grant 2015".

\section{References}

[1]. E. Wassermann, Oxford handbook of Transcranial Stimulation, Oxford University Press, Oxford (2007).

[2] W. Y. Kim, J. Magn. 19, 197 (2014).

[3] V. Walsh and A. Pascual-Leone, Transcranial magnetic stimulation: a neurochronometrics of mind, The MIT Press, Cambridge (2005).

[4] S.-S. Choi, Journal of Biomedicine and Biotechnology 278062 (2011).

[5] Mark S. George, Transcranial magnetic stimulation in clinical psychiatry. American psychiatric publishing inc (2007).

[6] J. H. Kim, J. Magn. 18, 20 (2014).

[7] V. Walsh, and A. Pascual-Leone, Transcranial magnetic stimulation: a neurochronometrics of mind. Cambridge, MA: MIT Press (2005).

[8] Carl Senior, Method in Mind, The MIT Press (2011).

[9] H. M. Koo, J. Magn. 19, 357 (2014).

[10] C. Edward Coffey and Jeffrey L. Cummings, The American Psychiatric Publishing Textbook of Geriatric Neuropsychiatry, Americna Psychiatric Press (2011).

[11] M. Sommer, N. Lang, F. Tergau, and W. Paulus, Neuroreport 13, 809 (2002). 